\title{
Isoproterenol enhances force production in mouse glycolytic and oxidative muscle via separate mechanisms
}

\author{
Sarah J. Blackwood ${ }^{1} \cdot$ Abram Katz $^{1}$ (D) \\ Received: 22 July 2019 / Revised: 22 July 2019 / Accepted: 13 August 2019 / Published online: 21 August 2019 \\ (C) The Author(s) 2019
}

\begin{abstract}
Fight or flight is a biologic phenomenon that involves activation of $\beta$-adrenoceptors in skeletal muscle. However, how force generation is enhanced through adrenergic activation in different muscle types is not fully understood. We studied the effects of isoproterenol (ISO, $\beta$-receptor agonist) on force generation and energy metabolism in isolated mouse soleus (SOL, oxidative) and extensor digitorum longus (EDL, glycolytic) muscles. Muscles were stimulated with isometric tetanic contractions and analyzed for metabolites and phosphorylase activity. Under conditions of maximal force production, ISO enhanced force generation markedly more in SOL (22\%) than in EDL (8\%). Similarly, during a prolonged tetanic contraction (30 s for SOL and $10 \mathrm{~s}$ for EDL), ISO-enhanced the force $\times$ time integral more in SOL (25\%) than in EDL (3\%). ISO induced marked activation of phosphorylase in both muscles in the basal state, which was associated with glycogenolysis (less in SOL than in EDL), and in EDL only, a significant decrease $(16 \%)$ in inorganic phosphate $\left(\mathrm{P}_{\mathrm{i}}\right)$. ATP turnover during sustained contractions $(1 \mathrm{~s}$ EDL, $5 \mathrm{~s}$ SOL) was not affected by ISO in EDL, but essentially doubled in SOL. Under conditions of maximal stimulation, ISO has a minor effect on force generation in EDL that is associated with a decrease in $\mathrm{P}_{\mathrm{i}}$, whereas ISO has a marked effect on force generation in SOL that is associated with an increase in ATP turnover. Thus, phosphorylase functions as a phosphate trap in ISOmediated force enhancement in EDL and as a catalyzer of ATP supply in SOL.
\end{abstract}

Keywords Isoproterenol $\cdot$ Muscle $\cdot$ Force $\cdot$ Fatigue $\cdot$ Metabolites $\cdot$ Phosphorylase

\section{Introduction}

Fight or flight is an established biologic response to a potentially harmful event or perceived threat to survival [16]. It entails a sympathetic discharge consisting of adrenaline (AD) release from the adrenal medulla (as well as noradrenaline from sympathetic nerves) that affects different organs and pathways in the body. With respect to skeletal muscle, AD binds to $\beta$-adrenoceptors and enhances force production in skeletal muscle. Inhibition of $\beta$-adrenoceptors (e.g., with propranolol) abolishes the effects of $\mathrm{AD}$ and sympathomimetics on muscle function $[9,19,29,31,60]$. Indeed, sympathomimetic agents increase force generation specifically via $\beta_{2}$ receptors [9]. The mechanism by which sympathomimetic agents enhance muscle performance has not been fully

Abram Katz

abram.katz@gih.se

1 Åstrand Laboratory of Work Physiology, Swedish School of Sport and Health Sciences, GIH, Box 5626, S-114 86 Stockholm, Sweden elucidated $[9,11,39]$, but evidence indicates that force augmentation during tetanic contractions occurs via increases in myoplasmic-free $\mathrm{Ca}^{2+}$ concentration $\left(\left[\mathrm{Ca}^{2+}\right]_{\mathrm{i}}\right)[3,11,15,30]$. The latter depends on cyclic AMP-dependent protein kinase (PKA)-mediated phosphorylation of ryanodine receptor 1 (serine residue S2844), which results in $\mathrm{Ca}^{2+}$ release from the sarcoplasmic reticulum (SR) [3].

Often, fight or flight lasts between several and $\sim 30 \mathrm{~s}$. Under such conditions, it appears that $\beta_{2}$-adrenergic activation induces a delay in skeletal muscle fatigue [14, 35, 39]. However, the mechanisms responsible for the enhancement of muscle force generation under such conditions are not fully understood, nor is it known whether the mechanisms are the same in different muscle fiber types. For example, adrenaline activates phosphorylase and stimulates glycogenolysis (albeit to a low extent compared with exercise) to a greater extent in glycolytic (primarily type II) vs. oxidative (primarily type I) muscle fibers at rest $[17,28,57]$. This would be expected to result in a greater accumulation of hexose monophosphates and a larger decrease in inorganic phosphate $\left(\mathrm{P}_{\mathrm{i}}\right)$ in glycolytic fibers. Since $P_{i}$ inhibits cross-bridge function $[2,21,50]$, one 
would expect this mechanism to be more prominent in enhancing muscle performance in glycolytic fibers. Thus, the purpose of the present study was to assess the effects of isoproterenol (ISO, $\beta$-adrenoceptor agonist) on force production, force $\times$ time integral, and metabolism in isolated mouse soleus (SOL, oxidative) and extensor digitorum longus (EDL, glycolytic) muscles under stimulation conditions that result in maximal force generation. Isolated muscles were studied to avoid confounding effects of other factors that could affect muscle function (e.g., changes in blood flow). The results indicate that under conditions of maximal stimulation, ISO enhances force production and force $\times$ time integral in both SOL and EDL, but more in SOL. The increased force in SOL is associated with an increased ATP turnover, whereas the increased force in EDL is associated with a decrease in the muscle content of $\mathrm{P}_{\mathrm{i}}$.

\section{Materials and methods}

\section{Animals and materials}

Adult male mice (C57Bl/6JOlaHSd, $n=50)$ aged 10 14 weeks $(\sim 25 \mathrm{~g})$ were housed at room temperature $\left(22{ }^{\circ} \mathrm{C}\right)$ on a 12:12-h light-dark cycle. Food and water were provided ad libitum. Animals were killed by cervical dislocation, and EDL and SOL muscles were isolated. Previous studies have established that EDL muscles of C57B16 mice express only fast myosin isoforms ( $\sim 80 \% \mathrm{IIb}, \sim 20 \% \mathrm{IIx})$, while SOL muscles express slow $(\sim 40 \% \mathrm{I})$ and fast $(\sim 55 \%$ IIa; $\sim 5 \%$ IIx $)$ myosin isoforms $[40,46]$. These myosin distributions indicate that EDL muscle fibers are primarily glycolytic, whereas the SOL muscle fibers are primarily oxidative [52]. The observation that the maximal mitochondrial respiration rate of saponin-skinned mouse soleus fibers is approximately twofold that of EDL fibers supports this conclusion [44]. All experiments were conducted in accordance with the Guide for the Care and Use of Laboratory Animals and were approved by the Ariel University animal ethics committee. All reagents, (-)-isoproterenol hydrochloride (ISO), and enzymes were from Sigma or Roche, except for D-[U- $\left.{ }^{14} \mathrm{C}\right]$-glucose-1-P and uridine diphosphate (UDP)-[U- $\left.{ }^{14} \mathrm{C}\right]$-glucose, which were purchased from Perkin-Elmer.

\section{Mounting, solution, and stimulation}

For stimulation experiments, silk sutures were tied to tendons and muscles mounted in a stimulation chamber (World Precision Instruments, FL, USA). One tendon was attached to a fixed hook and the other to a force transducer with an adjustable holder (allowing alteration of muscle length). Muscles were bathed in a Tyrode solution containing (in
$\mathrm{mM}): 121 \mathrm{NaCl}, 5 \mathrm{KCl}, 1.8 \mathrm{CaCl}_{2}, 0.5 \mathrm{NaH}_{2} \mathrm{PO}_{4}, 0.4$ $\mathrm{MgCl}_{2}, 24 \mathrm{NaHCO}_{3}, 0.1$ EDTA, and 5.5 glucose and constantly gassed with $5 \% \mathrm{CO}_{2}: 95 \% \mathrm{O}_{2}$, giving a final $\mathrm{pH}$ of 7.4. Chamber temperature was constantly maintained at $30{ }^{\circ} \mathrm{C}$ by a water jacketed circulation bath [61]. Muscles were stimulated electrically with platinum electrodes lying parallel to muscles using current pulses of $0.5 \mathrm{~ms}$ duration $(150 \%$ of the current required to elicit maximal force). After mounting, muscles were allowed to recover from dissection for $10 \mathrm{~min}$, and optimal length for generation of maximal isometric tetanic force was set with fused tetanic isometric contractions $(100 \mathrm{~ms}$ trains at $70 \mathrm{~Hz}$ ).

\section{Protocol $1-$ force frequency curves and continuous contraction}

After an equilibration period ( $25 \mathrm{~min} \mathrm{SOL}$ and $20 \mathrm{~min}$ EDL), a force frequency curve was performed followed by one sustained contraction (see below). Paired SOL and EDL muscles were stimulated at 1-min intervals using $600 \mathrm{~ms}$ (SOL, 1$100 \mathrm{~Hz})$ or $300 \mathrm{~ms}(\mathrm{EDL}, 1-150 \mathrm{~Hz})$ trains. Muscles then rested for $15 \mathrm{~min}$ and were subsequently stimulated to perform one sustained contraction (SOL, $100 \mathrm{~Hz}, 30 \mathrm{~s}$; EDL $120 \mathrm{~Hz}$, $10 \mathrm{~s}$ ). Thereafter, muscles recovered for $30 \mathrm{~min}$ either in the presence of $10 \mu \mathrm{M}$ ISO (diluted in dimethyl sulfoxide, DMSO) or in an equivalent volume of DMSO as a control $(\mathrm{CON}$; final concentration $=0.04 \%)$. Force frequency curves and sustained contractions were then repeated (see above) in the presence or absence of ISO. The ISO concentration and incubation duration employed should yield maximal effects $[24,38,56]$. The protocol is presented in schematic form in Fig. 1.

\section{Protocol 2 - EDL force frequency curve}

Protocol 1 produced a rundown effect in the EDL muscles, with CON treatment values lower at a number of contraction frequencies compared to baseline (Fig. 2c), as has been previously reported at $24-27{ }^{\circ} \mathrm{C}$ in fast-twitch muscle preparations $[13,56]$. Force frequency curves were therefore repeated on paired EDL muscles (before and after addition of ISO or DMSO) without a sustained contraction, as described above.

\section{Protocol 3 - stimulation for biochemical analysis}

Paired EDL and SOL were allowed to recover for 20 (EDL) or 25 (SOL) min after setting optimal length. Thereafter, and following a single control tetanus $(600 \mathrm{~ms}$ train, $100 \mathrm{~Hz}$ for SOL; $300 \mathrm{~ms}$ train $120 \mathrm{~Hz}$ for EDL), ISO or DMSO (CON) were added to the medium for $30 \mathrm{~min}$ (see above). In initial experiments, $\mathrm{NaCN}$ (final concentration $=3 \mathrm{mM}$ ) was added to the medium after $25 \mathrm{~min}$ of incubation in ISO or DMSO (to 


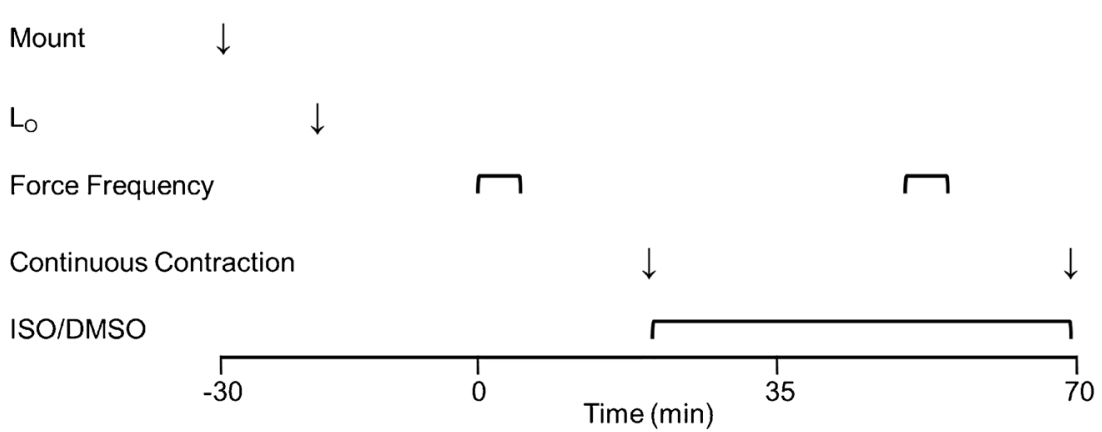

Fig. 1 Scheme for Protocol 1. After setting optimal length $\left(\mathrm{L}_{\mathrm{O}}\right)$, EDL muscles equilibrated for $20 \mathrm{~min}$, followed by baseline force frequency and a prolonged (continuous) contraction. Thereafter, ISO (or DMSO)

was administered for $30 \mathrm{~min}$ before force frequency and continuous contraction was repeated. The scheme looks the same for soleus muscles with the exception that after setting $\mathrm{L}_{\mathrm{o}}$, equilibration was for $25 \mathrm{~min}$

inhibit cytochrome oxidase to create an anoxic environment, thereby enabling estimates of ATP turnover). In subsequent experiments, $\mathrm{NaCN}$ was omitted (see "Results"). Muscles were then stimulated to perform one sustained contraction (as in protocol 1) for 1 (EDL) or $5 \mathrm{~s}$ (SOL) and the muscles were subsequently rapidly frozen in liquid $\mathrm{N}_{2}$. Non-stimulated controls were frozen at the same times of incubation. The intent of these experiments was to correlate metabolic changes with the enhancement of force by ISO.

\section{Analytical}

Force signals were recorded online with LabScribe2 software (iWorx, NH, USA) and stored on a personal computer for subsequent analysis. Peak tension was measured during tetani. Time of force development and relaxation time were estimated as time to $50 \%$ of peak tension ( $1 / 2$ TTP) and time to $50 \%$ relaxation ( $1 / 2$ RT) [43]. For analytical biochemistry, muscles were freeze-dried and freed from non-muscle constituents. Muscle tissue was powdered, thoroughly mixed, and processed further (for glycogen) or divided into two aliquots (for enzymes and metabolites). One aliquot was extracted in icecold $0.5 \mathrm{M}$ perchloric acid, centrifuged, neutralized with 2.2 $\mathrm{KHCO}_{3}$, and centrifuged again. The final supernatant was analyzed for metabolites using enzymatic techniques (changes in $\mathrm{NAD}[\mathrm{P}] \mathrm{H})$ adapted for fluorometry [45]. To correct for variability in solid non-muscle constituents, metabolite values were adjusted for total creatine (TCr). ISO did not significantly affect TCr under any condition studied. TCr averaged 73.8 $\pm 1.6 \mu \mathrm{mol} / \mathrm{g}$ dry weight in SOL and $128.8 \pm 2.4 \mu \mathrm{mol} / \mathrm{g}$ dry weight in EDL. For analysis of glycogen, muscle was digested in hot $\left(80^{\circ} \mathrm{C}\right) 1 \mathrm{~N} \mathrm{NaOH}$ and hydrolyzed with amyloglucosidase to free glucose [42], which was converted to NADPH and analyzed fluorometrically [45]. Aliquots of extract were analyzed for protein (BioRad method) and glycogen values were adjusted for protein. ISO did not significantly affect protein under any condition studied and protein averaged $357 \pm 5 \mu \mathrm{g} / \mathrm{mg}$ dry weight in EDL and $326 \pm 7 \mu \mathrm{g} /$ mg dry weight in SOL.

Anaerobic ATP turnover was calculated as follows: $2 \Delta \mathrm{ATP}-\Delta \mathrm{PCr}+(1.5 \Delta$ lactate $)[42]$, where $\Delta$ is the mean contraction value minus the mean basal value (Table 3 ). In using this formula, it is assumed that during short-term intense contractions all lactate production is from glycogen and there is no loss of lactate into the medium [42]. In experiments where $\mathrm{NaCN}$ was omitted, aerobic ATP production during contraction was estimated using published $\mathrm{O}_{2}$ consumption values of isolated mouse SOL $(4 \mu \mathrm{L} / \mathrm{s} / \mathrm{g}$ wet weight $)$ and $\operatorname{EDL}(13.5 \mu \mathrm{L} /$ $\mathrm{s} / \mathrm{g}$ wet weight) [5] (adjusting for temperature differences by assuming a $\mathrm{Q}_{10}$ of 2) and myoglobin concentration of oxidative $(3.15 \mathrm{mg} / \mathrm{g}$ wet weight $)$ and glycolytic $(0.5 \mathrm{mg} / \mathrm{g}$ wet weight) rodent skeletal muscle [33], assuming a myoglobin molecular mass of $16.95 \mathrm{kDa}$ and a myoglobin: $\mathrm{O}_{2}$ ratio of 1:1, a $\mathrm{P} / \mathrm{O}_{2}$ of 6 and $4.3 \mathrm{~g}$ wet weight $/ \mathrm{g}$ dry weight.

The second aliquot was homogenized $(200 \mu \mathrm{L} / \mathrm{mg}$ dry weight) with a ground glass homogenizer in an ice-cold buffer of (in mM): 10 EDTA, $50 \mathrm{KF}$, and 30\% glycerol (v/v), $\mathrm{pH} 7.0$, and centrifuged. The supernatant was diluted and used for analysis of glycogen phosphorylase (Phos) and glycogen synthase (GS) activity following the incorporation of D- $\left[\mathrm{U}-{ }^{14} \mathrm{C}\right] \mathrm{glucose}-1-\mathrm{P}$ into glycogen and UDP-[U- $\left.{ }^{14} \mathrm{C}\right]$ glucose into glycogen, respectively, as previously described. Total and fractional activities ( $\pm 3 \mathrm{mM}$ AMP for Phos; 0.17/7.2 mM G6P for GS) were measured as described elsewhere [42], with the exception that glucose 1-P concentration was $67 \mathrm{mM}$ in the absence and presence of AMP. Total activities were adjusted for $\mathrm{TCr}$ as above.

\section{Statistics}

Values are given as mean \pm SE. Statistical significance was set at $P<0.05$ and was determined with paired or unpaired $t$ tests. Results from muscles that produced low forces were excluded. 
Fig. 2 Isoproterenol enhances force generation of soleus and extensor digitorum longus muscles. Force frequency curves were performed at baseline $(\circ, \square)$ and $30 \mathrm{~min}$ post-treatment for control $(\bullet)$ and isoproterenol ( $(\boldsymbol{\bullet})$ groups in soleus $(\mathrm{SOL})(\mathbf{a}, \mathbf{b})$ and extensor digitorum longus (EDL) $(\mathbf{c}-\mathbf{f})$, respectively. Forces are substantially higher than baseline in the presence of ISO in SOL (b). After baseline, a prolonged contraction was performed in each muscle (see Fig. 3), which caused a rundown effect in the EDL muscle with CON values significantly decreasing from baseline (c). Consequently, there were only minor increases in force in the presence of ISO (d). Force frequency curves were therefore performed in another series of EDL muscles without continuous contraction after baseline, CON (e) and ISO (f). In the latter case, forces are clearly higher than baseline in the presence of ISO (f). The corresponding average percentage effect of ISO on force is shown for $\mathrm{SOL}(\mathbf{g})$ and $\mathrm{EDL}(\mathbf{h})$ muscles. $\mathbf{h}$ is derived from results from $\mathbf{d}$ and $\mathbf{f}$, with adjustments for rundown in $\mathbf{d}$. Data are expressed as mean \pm SE for $5\left(\mathbf{e}^{-}-\mathbf{f}\right), 8(\mathbf{a}-\mathbf{d}$ and g), or 13 (h) muscles.

$* p<0.05 ; * * p<0.01$;

$* * * p<0.001$ vs. corresponding

treatment by paired $t$ test
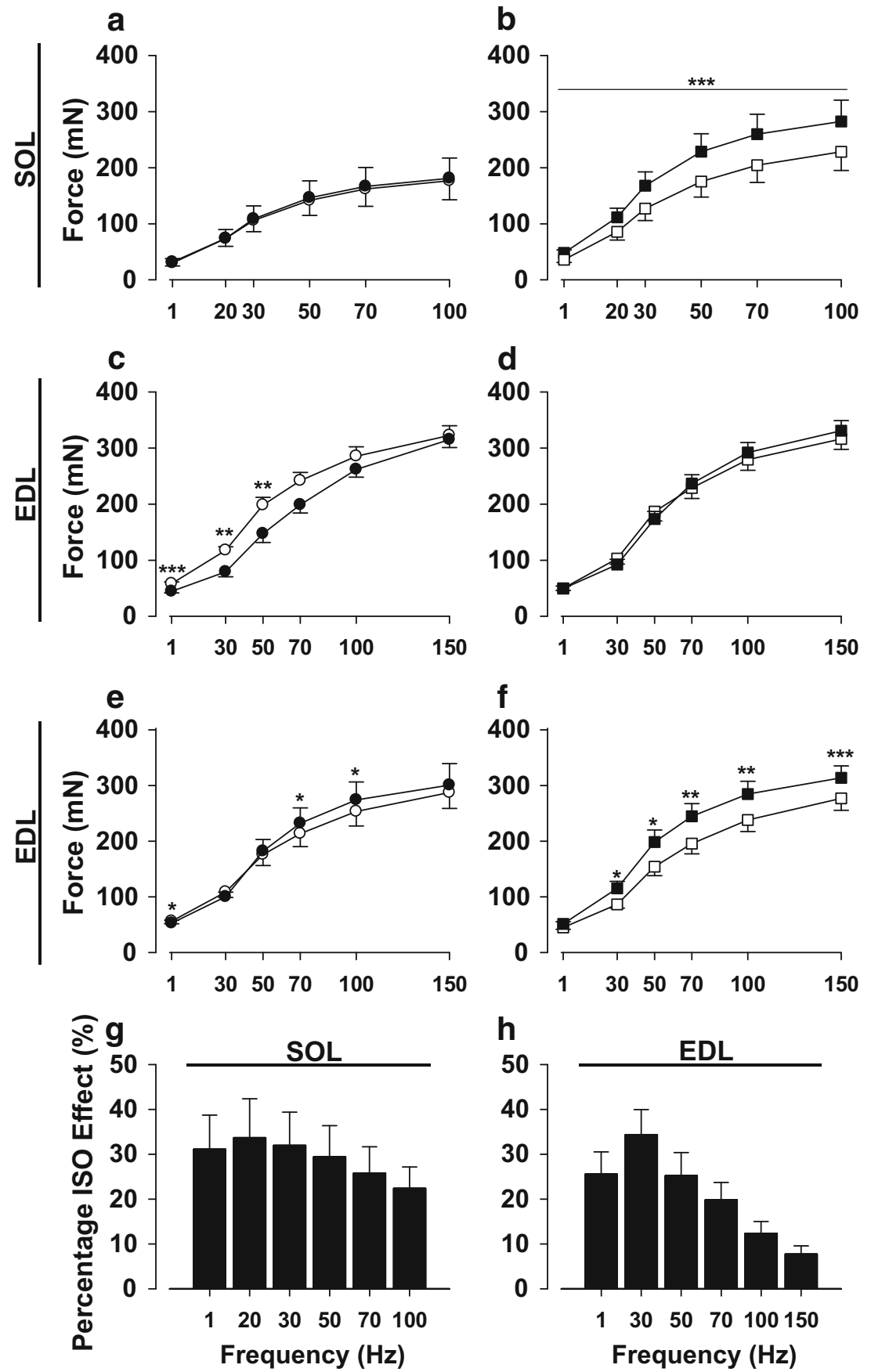

\section{Results}

\section{Effects of isoproterenol on contractile properties of SOL and EDL muscles}

Exposure to ISO-enhanced force generation in SOL muscles at all stimulation frequencies, while similar force values were obtained before and after addition of DMSO (Fig. 2a, b). In contrast, ISO resulted in increased forces only at high stimulation frequencies in EDL (Fig. 2d); however, a rundown effect was noted in the control group (decrease in force during second force frequency run), especially at the lower stimulation frequencies (Fig. 2c). Therefore, an additional series of experiments was performed where prolonged continuous tetani were omitted. Under these conditions, rundown was abolished and an ISO effect on force was seen at all stimulation frequencies (Fig. 2e and f). Estimates of ISO-mediated force enhancement showed a robust effect in SOL at all stimulation frequencies (Fig. 1g). In the EDL, force enhancement was substantial at lower frequencies but less so at the higher frequencies (Fig. 1h). Thus, the peak ISO effect occurred at low frequencies in both SOL $(33.7 \pm 8.7 \%$ at $20 \mathrm{~Hz}$, Fig. $1 \mathrm{~g})$ 
and EDL $(34.4 \pm 5.6 \%$ at $30 \mathrm{~Hz}$, Fig. $1 \mathrm{H})$, whereas for maximal tetanic contractions, the ISO effect was better maintained in the SOL $(22.4 \pm 4.8 \%$ at $100 \mathrm{~Hz})$ than in the EDL $(7.7 \pm$ $1.8 \%$ at $150 \mathrm{~Hz})(P<0.01$ for SOL vs. EDL). The latter is clearly seen in representative force recordings for both EDL and soleus muscles (Fig. 3). The $1 / 2$ time to peak tension (TTP) was slightly faster after ISO treatment in SOL at the higher frequencies, but a similar observation was observed in the control condition at $100 \mathrm{~Hz}$ (treatment with DMSO vs. baseline). Therefore, these changes are not considered to be of physiologic significance (Table 1).

\section{Effects of isoproterenol on force $x$ time integral in SOL and EDL muscles}

Representative traces in Fig. 4 show similar force production during continuous stimulations 30 min apart in CON groups for both SOL (4A) and EDL (4D). In SOL, ISO markedly enhanced force output and this was maintained throughout the $30 \mathrm{~s}$ tetanus (Fig. 4b). In EDL, ISO had a minor effect on force generation that was observed primarily at the onset of contraction (Fig. 4e). The force $\times$ time integral during contraction was markedly enhanced by ISO in SOL $(25.4 \pm 4.9 \%$; Fig. 4c), but only slightly in EDL $(3.2 \pm 1.3 \%$; Fig. 4 f) $(P<0.01$ for SOL vs. EDL $)$.

\section{ISO and metabolism SOL and EDL muscles}

To assess whether the enhancement of force generation during continuous contractions could be attributed to cellular metabolic changes, muscles were frozen at $1 \mathrm{~s}$ (EDL) or $5 \mathrm{~s}(\mathrm{SOL})$ of continuous contractions (time points where force was elevated - Fig. 4). Initial experiments were performed in the presence of sodium cyanide $(\mathrm{NaCN})$ to allow for assessment of ATP turnover [42, 61]. Following $30 \mathrm{~min}$ exposure to ISO, phosphorylase fractional activity increased to $\sim 30 \%$ in SOL and to $\sim 55 \%$ in EDL muscles at rest in the presence of $\mathrm{NaCN}$ (Fig. 5a, b). Following a single sustained contraction under control conditions in the presence of $\mathrm{NaCN}$, fractional activity increased to almost $70 \%$ in SOL and $80 \%$ in EDL, and ISO had no additional effect. As expected, ISO resulted in marked decreases in glycogen synthase (GS) fractional activity in SOL and EDL at rest in the absence or presence of $\mathrm{NaCN}$ (Fig. 5c, d). Contraction resulted in marked decreases of GS fractional activity as well, with no further effect of ISO in either muscle.

Basal state measurements of metabolites after $5 \mathrm{~min}$ in $\mathrm{NaCN}$ showed a marked depletion of phosphocreatine (PCr, $\sim 30 \%$ in SOL and 60\% in EDL) and accumulation of lactate ( $\sim$-fold in SOL and 13-fold in EDL) (data not shown). These changes in metabolite values precluded accurate measurements of energy turnover. Similar results were previously
Fig. 3 Representative force recordings for soleus and extensor digitorum longus muscles during maximal stimulation frequencies. Forces are shown for a given muscle before (solid line) and after (dashed line) treatment. Soleus muscles were stimulated at $100 \mathrm{~Hz}$ before and after exposure to diluent (a) or ISO (b). Extensor digitorum longus (EDL) muscles were stimulated at $150 \mathrm{~Hz}$ before and after exposure to diluent (c) or ISO (d). Note that at these frequencies ISO enhanced force markedly more in soleus than in EDL
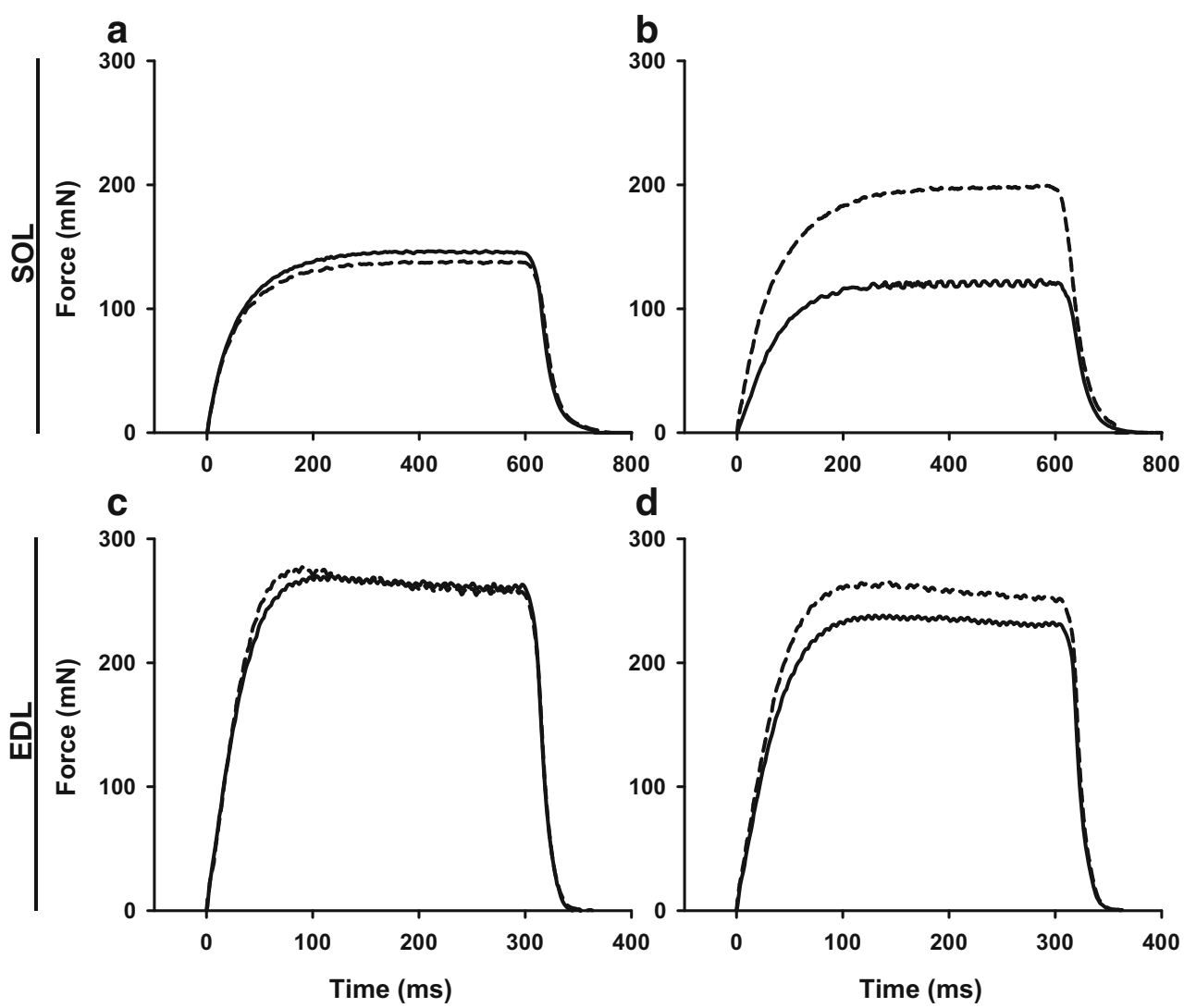
Table 1 Effects of isoproterenol on contractile properties in soleus and extensor digitorum longus muscles

\begin{tabular}{|c|c|c|c|c|c|c|c|c|c|}
\hline \multicolumn{2}{|c|}{ Stimulation frequency } & \multicolumn{4}{|c|}{ Time to $50 \%$ of peak tension $(\mathrm{ms})$} & \multicolumn{4}{|c|}{ Time to $50 \%$ of decrease of tension (ms) } \\
\hline & & \multicolumn{2}{|l|}{ Control } & \multicolumn{2}{|c|}{ Isoproterenol } & \multicolumn{2}{|l|}{ Control } & \multicolumn{2}{|c|}{ Isoproterenol } \\
\hline & & Baseline & Treatment & Baseline & Treatment & Baseline & Treatment & Baseline & Treatment \\
\hline \multirow[t]{6}{*}{ SOL } & $1 \mathrm{~Hz}$ & $6.6 \pm 0.2$ & $6.6 \pm 0.4$ & $5.9 \pm 0.2$ & $6.4 \pm 0.2$ & $37.9 \pm 3.6$ & $33.0 \pm 1.7$ & $31.0 \pm 1.5$ & $29.6 \pm 1.2$ \\
\hline & $20 \mathrm{~Hz}$ & $54.0 \pm 4.9$ & $57.3 \pm 0.6$ & $47.5 \pm 7.4$ & $47.9 \pm 7.0$ & $44.8 \pm 1.8$ & $45.8 \pm 2.7$ & $47.8 \pm 3.4$ & $43.1 \pm 2.7^{*}$ \\
\hline & $30 \mathrm{~Hz}$ & $56.9 \pm 4.4$ & $53.5 \pm 4.5$ & $60.5 \pm 4.9$ & $57.0 \pm 4.8$ & $42.6 \pm 1.6$ & $43.0 \pm 1.3$ & $43.8 \pm 4.0$ & $42.1 \pm 1.4$ \\
\hline & $50 \mathrm{~Hz}$ & $55.0 \pm 2.1$ & $53.0 \pm 2.5$ & $52.9 \pm 2.6$ & $50.6 \pm 2.0 *$ & $41.3 \pm 1.2$ & $44.8 \pm 2.2$ & $42.3 \pm 2.0$ & $39.6 \pm 1.1$ \\
\hline & $70 \mathrm{~Hz}$ & $51.1 \pm 2.0$ & $49.8 \pm 1.9$ & $49.5 \pm 1.8$ & $46.9 \pm 1.7 *$ & $41.5 \pm 1.3$ & $42.9 \pm 1.7$ & $41.1 \pm 2.5$ & $40.9 \pm 1.3$ \\
\hline & $100 \mathrm{~Hz}$ & $46.9 \pm 1.8$ & $44.9 \pm 1.4^{*}$ & $44.9 \pm 1.7$ & $42.8 \pm 1.6^{* *}$ & $44.5 \pm 1.6$ & $46.6 \pm 2.2$ & $46.0 \pm 2.1$ & $45.0 \pm 1.7$ \\
\hline \multirow[t]{6}{*}{ EDL } & $1 \mathrm{~Hz}$ & $5.0 \pm 0.3$ & $4.6 \pm 0.2$ & $4.2 \pm 0.2$ & $4.4 \pm 0.2$ & $24.6 \pm 1.3$ & $20.0 \pm 2.7$ & $24.8 \pm 1.2$ & $21.6 \pm 2.1$ \\
\hline & $30 \mathrm{~Hz}$ & $16.0 \pm 5.3$ & $20.4 \pm 6.4$ & $10.8 \pm 0.8$ & $22.4 \pm 6.2$ & $14.4 \pm 0.7$ & $12.4 \pm 1.4$ & $14.2 \pm 1.5$ & $12.8 \pm 0.7$ \\
\hline & $50 \mathrm{~Hz}$ & $25.8 \pm 0.5$ & $28.0 \pm 1.7$ & $29.6 \pm 3.1$ & $31.6 \pm 3.0$ & $15.4 \pm 1.2$ & $15.4 \pm 1.9$ & $14.4 \pm 1.4$ & $18.0 \pm 1.7$ \\
\hline & $70 \mathrm{~Hz}$ & $25.8 \pm 2.0$ & $28.0 \pm 2.5$ & $31.0 \pm 0.7$ & $32.0 \pm 0.9$ & $16.2 \pm 0.6$ & $18.0 \pm 1.0$ & $15.8 \pm 0.7$ & $18.6 \pm 1.4^{*}$ \\
\hline & $100 \mathrm{~Hz}$ & $25.0 \pm 1.1$ & $26.0 \pm 1.8$ & $27.8 \pm 1.7$ & $28.2 \pm 1.2$ & $17.8 \pm 0.7$ & $19.6 \pm 0.7 * *$ & $16.6 \pm 0.7$ & $19.0 \pm 0.9$ \\
\hline & $150 \mathrm{~Hz}$ & $22.6 \pm 1.0$ & $23.6 \pm 1.2$ & $24.8 \pm 1.4$ & $24.8 \pm 0.8$ & $19.6 \pm 0.9$ & $20.8 \pm 1.0^{*}$ & $20.2 \pm 1.0$ & $22.4 \pm 3.0$ \\
\hline
\end{tabular}

Results are obtained from force frequency curves where solei (SOL) were stimulated with a single 600-ms train and extensor digitorum longus (EDL) muscles were stimulated with a single 300-ms train. Data are expressed as mean $\pm \mathrm{SE}$ for five (EDL, no rundown muscles) or eight (SOL) muscles

$* p<0.05$

$* * p<0.01$ vs. corresponding baseline value by paired $t$ test
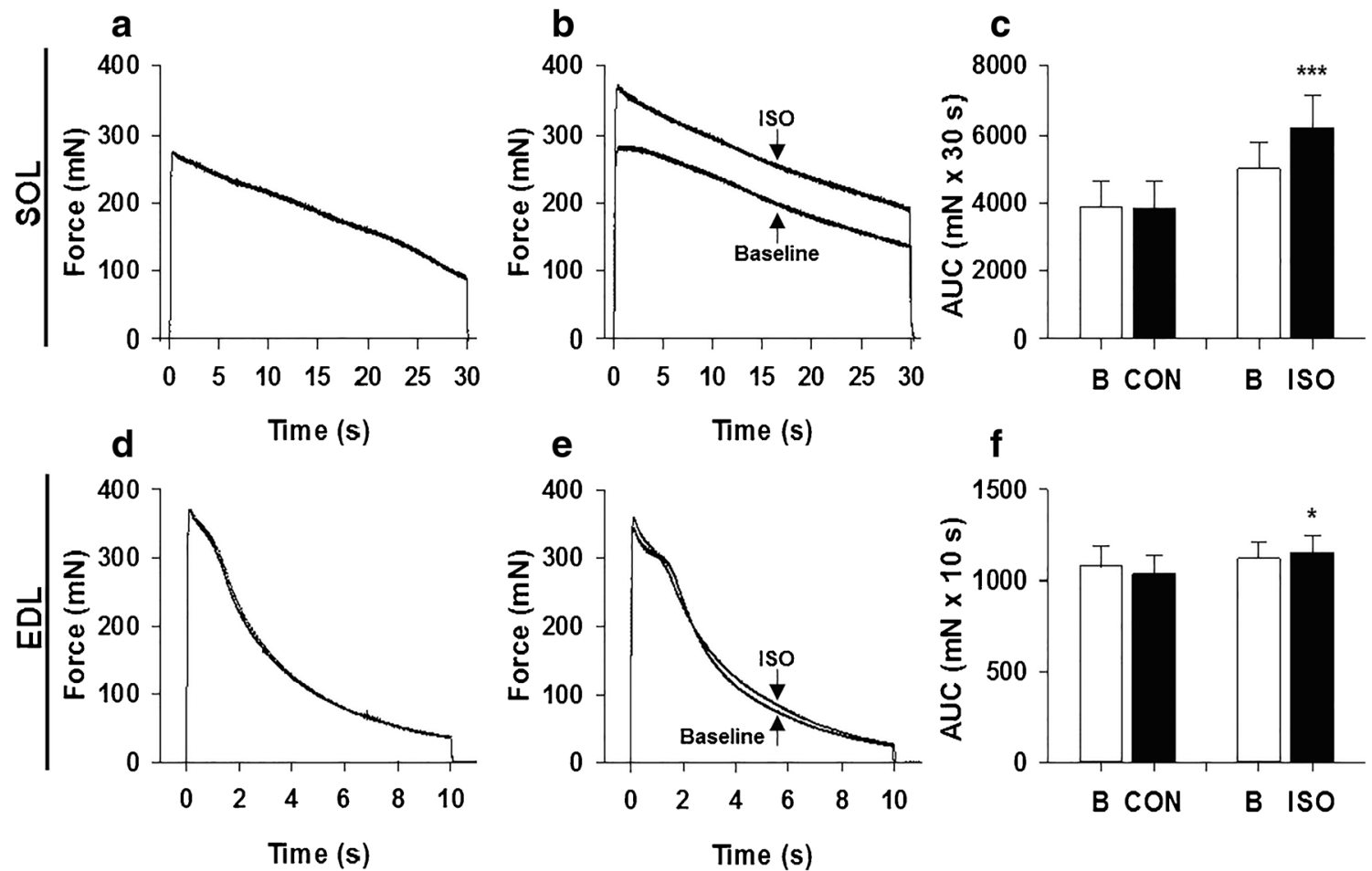

Fig. 4 Isoproterenol enhances work output during prolonged contractions of soleus and extensor digitorum longus muscles. Prolonged tetanic contractions were performed for $30 \mathrm{~s}$ at a frequency of $100 \mathrm{~Hz}$ in SOL and $10 \mathrm{~s}$ at a frequency of $120 \mathrm{~Hz}$ in EDL. Representative traces are shown for baseline and $30 \mathrm{~min}$ post-treatment in CON and ISO groups as indicated for SOL $(\mathbf{a}, \mathbf{b})$ and $\operatorname{EDL}(\mathbf{d}, \mathbf{e})$, respectively. Note that in a and $\mathbf{d}$, baseline and CON curves are virtually identical, whereas in panel $\mathbf{b}$ the ISO curve is markedly higher throughout

in comparison to baseline and in e the ISO curve is slightly higher initially vs. baseline. The effect of ISO on force $\times$ time integral was quantified by determining the area under the curve (AUC) of the continuous contractions in SOL (c) and EDL (f) muscles at baseline (B, unfilled bar) and 30 min post-treatment (CON and ISO, filled bars). Data are expressed as mean \pm SE for eight muscles. $* p<0.05 ; * * *<0.001$ vs. corresponding baseline values by paired $t$ test 
Fig. 5 Isoproterenol activates phosphorylase and inactivates glycogen synthase in soleus and extensor digitorum longus muscles. Phosphorylase (Phos) and glycogen synthase (GS) fractional activities were determined in soleus (SOL, a and c) and extensor digitorum longus muscles (EDL, $\mathbf{b}$ and $\mathbf{d}$ ).

Treatments consisted of basal control (BC), basal isoproterenol (BI), stimulated control (SC), and stimulated isoproterenol (SI) in the absence $(-)$ or presence $(+)$ of $3 \mathrm{mM}$ sodium cyanide. SOL muscles were stimulated for $5 \mathrm{~s}$ at $100 \mathrm{~Hz}$ and EDL muscles for $1 \mathrm{~s}$ at $120 \mathrm{~Hz}$. Data are expressed as mean \pm SE for 5-7 muscles (numbers vary as sufficient extract was not always available). $* \mathrm{p}<0.05 ; * * p<0.01 ; * * * \mathrm{p}<$ 0.001 vs. corresponding baseline values by paired $t$ test

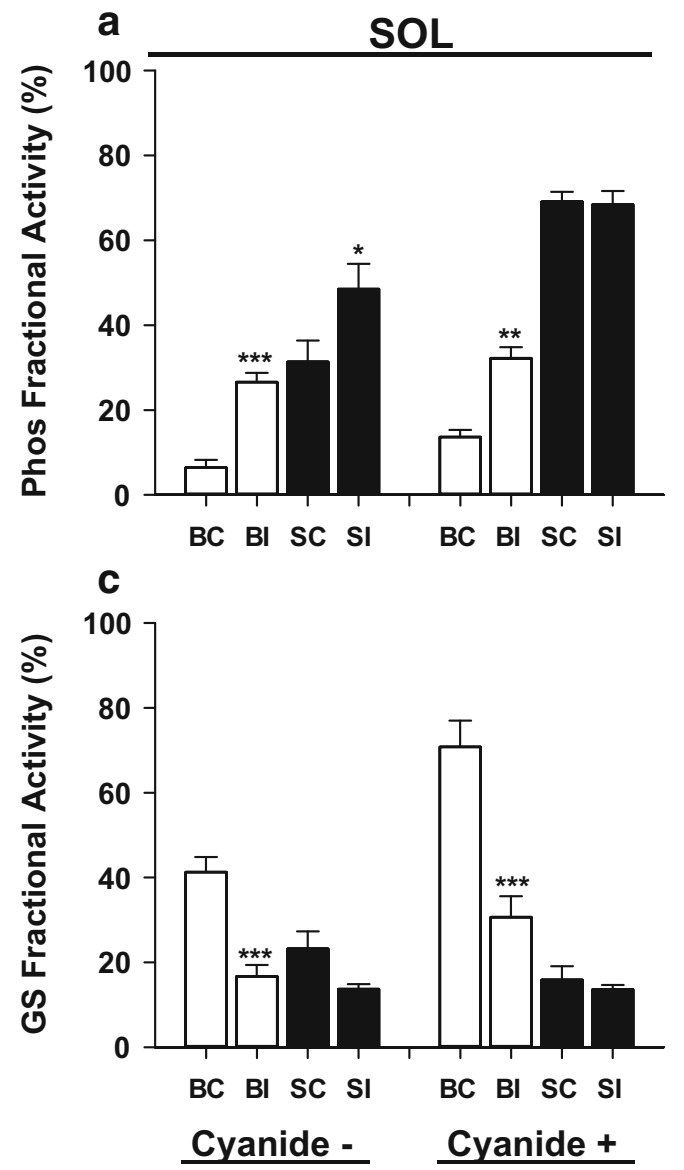

reported in isolated rat EDL muscle following 10 min incubation in $2 \mathrm{mM} \mathrm{NaCN}$ at $30{ }^{\circ} \mathrm{C} \mathrm{[54].} \mathrm{Therefore,} \mathrm{experiments}$ were repeated in the absence of $\mathrm{NaCN}$. With respect to enzyme activities, the results were similar to those observed in $\mathrm{NaCN}$, with some differences. First, basal phosphorylase fractional activity was $\sim 50 \%$ lower in SOL and EDL in the absence of $\mathrm{NaCN}$ (Fig. 4). This is likely explained by a small increase in $\left[\mathrm{Ca}^{2+}\right]_{\mathrm{i}}$ as a result of $\mathrm{NaCN}$ exposure, which should result in activation of phosphorylase kinase and thereby phosphorylation of phosphorylase b [41]. Second, the stimulation-mediated activation of phosphorylase in SOL was only about half that seen in the presence of $\mathrm{NaCN}$ (increase to $31 \%$ vs. $69 \%$ in $\mathrm{NaCN}$ ), which corresponded with an additive effect of ISO and stimulation on fractional activity. Last, GS fractional activity was markedly lower in SOL in the basal state in the absence of $\mathrm{NaCN}$. Total activities of phosphorylase and GS were not affected by ISO under any condition (Table 2).

In the basal state, exposure to ISO for 30 min resulted in a decrease of glycogen of almost $10 \mu \mathrm{mol}$ glucosyl units/g dry weight in SOL muscle, but this decrease was not statistically significant (Table 3 ). In the basal condition, ISO resulted in a $\sim 5$-fold increase in glucose 6-P, but no significant change in lactate. Accumulation of glucose 6-P and lactate accounted for $\sim 50 \%$ of glycogen breakdown induced by ISO, indicating that approximately half of the glycogenolytic breakdown products were either released (as lactate) or oxidized (as pyruvate) in mitochondria. It should be noted that in the presence of $\beta$-adrenergic stimulation, glucose transport is either unaffected or decreased in skeletal muscle $[8,18,60]$, indicating that the increase in glycogenolytic intermediates in the presence of ISO is derived from glycogen breakdown and not glucose transport. Other metabolites were not significantly affected by ISO at rest in SOL. However, following contraction, ISO resulted in significantly greater accumulation of lactate and larger depletion of PCr. In the EDL muscle under basal conditions, ISO resulted in a breakdown of $\sim 20 \mu \mathrm{mol}$ glucosyl units/g dry weight, a > 15-fold increase in glucose 6$\mathrm{P}$, and a significant increase in lactate (accumulation of glycogenolytic intermediates accounted for $<30 \%$ of glycogen breakdown), demonstrating a greater activation of glycogenolysis compared to SOL. Additionally, in EDL in the basal state, ISO resulted in a statistically significant decrease in $\mathrm{P}_{\mathrm{i}}$ (16\%). Following contraction, ISO did not significantly affect any of the measured metabolites in EDL. In the stimulated state, glycogen values in the presence of ISO did not differ significantly from control in either muscle. It should be noted, however, that under the present conditions ( 1 and $5 \mathrm{~s}$ contractions for EDL and SOL, respectively), accurate measurements of glycogenolysis are better reflected by accumulation of 
Table 2 Total phosphorylase and glycogen synthase activities in soleus and extensor digitorum longus muscles in the absence or presence of sodium cyanide

\begin{tabular}{|c|c|c|c|c|c|c|c|c|c|}
\hline & & \multicolumn{4}{|l|}{ Cyanide } & \multicolumn{4}{|l|}{ Cyanide + } \\
\hline & & \multicolumn{2}{|l|}{ Basal } & \multicolumn{2}{|l|}{ Stimulated } & \multicolumn{2}{|l|}{ Basal } & \multicolumn{2}{|l|}{ Stimulated } \\
\hline & & Control & Isoproterenol & Control & Isoproterenol & Control & Isoproterenol & Control & Isoproterenol \\
\hline \multirow[t]{2}{*}{ Phos } & $S O L$ & $69.1 \pm 4.1$ & $77.6 \pm 5.9$ & $77.9 \pm 6.5$ & $62.2 \pm 6.8$ & $94.8 \pm 7.2$ & $77.3 \pm 9.8$ & $89.8 \pm 10.6$ & $96.7 \pm 8.0$ \\
\hline & $E D L$ & $224.2 \pm 19.0$ & $227.2 \pm 20.5$ & $250.7 \pm 8.6$ & $293.5 \pm 25.7$ & $279.0 \pm 7.8$ & $265.0 \pm 11.0$ & $309.0 \pm 7.0$ & $312.0 \pm 33.2$ \\
\hline \multirow[t]{2}{*}{ GS } & $S O L$ & $3.3 \pm 0.4$ & $3.0 \pm 0.1$ & $3.5 \pm 0.5$ & $2.4 \pm 0.6$ & $2.6 \pm 0.3$ & $2.9 \pm 0.5$ & $3.1 \pm 0.2$ & $2.8 \pm 0.4$ \\
\hline & $E D L$ & $2.6 \pm 0.3$ & $1.9 \pm 0.3$ & $1.9 \pm 0.3$ & $1.6 \pm 0.4$ & $1.9 \pm 0.2$ & $1.9 \pm 0.2$ & $1.8 \pm 0.2$ & $1.4 \pm 0.2$ \\
\hline
\end{tabular}

Experimental conditions are as in the legend of Fig. 3. Data expressed as mean $\pm \mathrm{SE}$ and are given in $\mu \mathrm{mol} / \mathrm{min} / \mathrm{g}$ dry weight for 5-7 muscles (numbers vary as sufficient extract was not always available). Paired $t$ test used to determine significance

glycogenolytic intermediates (primarily glucose 6-P and lactate), as discussed elsewhere [42].

Estimates of non-aerobic ATP turnover during contraction in SOL averaged 11.5 and $29.2 \mu \mathrm{mol} / \mathrm{g}$ dry weight in the absence and presence of ISO, respectively, and total ATP turnover (including utilization of oxygen) averaged 22.2 and $39.3 \mu \mathrm{mol} / \mathrm{g}$ dry weight, respectively. Estimates of nonaerobic ATP turnover during contraction in EDL averaged 91.3 and $84.6 \mu \mathrm{mol} / \mathrm{g}$ dry weight in the absence and presence of ISO, respectively, and total ATP turnover (including utilization of oxygen) averaged 97.3 and $90.6 \mu \mathrm{mol} / \mathrm{g}$ dry weight, respectively. It was assumed that ISO did not affect oxygen consumption under any condition, and this was supported by the finding that malate (a marker of oxidative metabolism [36, 61]) was not affected by ISO under any condition (Table 3 ). The minor relative contribution of oxygen to total ATP turnover in EDL and significant contribution in SOL is consistent with previous findings under comparable conditions [22]. Thus, ISO markedly increased ATP turnover in SOL during contraction, but was without noteworthy effect in EDL.

\section{Discussion}

The major findings of the present study are that ISO: (1) increases maximal force production and force $\times$ time integral more in SOL than in EDL; (2) activates glycogenolysis in the basal state to a greater extent in EDL, but during contraction glycogenolysis is enhanced only in SOL; and (3) decreases $\mathrm{P}_{\mathrm{i}}$ in the basal state in EDL and increases ATP turnover during contraction in SOL.

In accordance with previous studies $[3,12,15,30]$, we observed that activation of $\beta$-adrenoceptors enhances force markedly in SOL and EDL during low stimulation

Table 3 Metabolite contents in soleus and extensor digitorum longus muscles

\begin{tabular}{|c|c|c|c|c|c|c|c|c|}
\hline & \multicolumn{4}{|l|}{$S O L$} & \multicolumn{4}{|l|}{$E D L$} \\
\hline & \multicolumn{2}{|l|}{ Basal } & \multicolumn{2}{|l|}{ Stimulated } & \multicolumn{2}{|l|}{ Basal } & \multicolumn{2}{|l|}{ Stimulated } \\
\hline & $\mathrm{CON}$ & ISO & $\mathrm{CON}$ & ISO & $\mathrm{CON}$ & ISO & $\mathrm{CON}$ & ISO \\
\hline Glycogen & $70.4 \pm 4.8$ & $61.6 \pm 5.8$ & $57.6 \pm 6.7$ & $56.1 \pm 7.9$ & $52.3 \pm 4.8$ & $32.5 \pm 1.8 * *$ & $29.3 \pm 3.7$ & $25.8 \pm 2.4$ \\
\hline$G 6 P$ & $0.71 \pm 0.09$ & $3.94 \pm 0.17 * * *$ & $3.75 \pm 0.50$ & $4.31 \pm 0.36$ & $0.22 \pm 0.04$ & $3.79 \pm 0.42 * * *$ & $5.93 \pm 0.92$ & $6.36 \pm 0.62$ \\
\hline Lactate & $6.3 \pm 1.0$ & $9.1 \pm 1.9$ & $12.5 \pm 1.0$ & $17.8 \pm 2.0 * *$ & $1.7 \pm 0.3$ & $5.1 \pm 0.4 * *$ & $28.5 \pm 5.4$ & $32.7 \pm 4.8$ \\
\hline Malate & $0.97 \pm 0.12$ & $1.00 \pm 0.07$ & $1.66 \pm 0.12$ & $1.91 \pm 0.17$ & $0.18 \pm 0.03$ & $0.26 \pm 0.04$ & $0.73 \pm 0.10$ & $0.94 \pm 0.11$ \\
\hline$A T P$ & $17.4 \pm 0.6$ & $17.7 \pm 1.6$ & $20.1 \pm 1.0$ & $17.1 \pm 0.7$ & $28.9 \pm 0.7$ & $27.8 \pm 0.5$ & $25.9 \pm 1.4$ & $27.9 \pm 2.4$ \\
\hline$P i$ & $26.1 \pm 2.2$ & $22.5 \pm 1.1$ & $34.9 \pm 2.2$ & $36.2 \pm 2.1$ & $16.9 \pm 0.8$ & $14.3 \pm 0.6^{*}$ & $47.8 \pm 6.3$ & $54.0 \pm 8.2$ \\
\hline$P C r$ & $40.9 \pm 1.9$ & $39.4 \pm 1.8$ & $33.2 \pm 3.1$ & $24.6 \pm 2.6^{* *}$ & $89.2 \pm 1.3$ & $87.6 \pm 1.5$ & $44.0 \pm 6.8$ & $44.2 \pm 4.2$ \\
\hline $\mathrm{Cr}$ & $28.6 \pm 1.9$ & $30.0 \pm 1.8$ & $36.1 \pm 3.1$ & $44.7 \pm 2.6^{* *}$ & $20.3 \pm 1.3$ & $21.8 \pm 1.5$ & $93.1 \pm 6.8$ & $85.3 \pm 8.4$ \\
\hline
\end{tabular}

Data are expressed as mean \pm SE. Values are given in $\mu \mathrm{mol} / \mathrm{g}$ dry weight (for glycogen $\mu \mathrm{mol}$ glucosyl units/g dry weight), six muscles for metabolites and seven (SOL) and six (EDL) glycogen

$* p<0.05$

$* * p<0.01$

$* * * p<0.001$ vs. CON by paired $t$ test 
frequencies. Further, we found that the $\beta$-adrenergic mediated enhancement of force during high stimulation frequency continues to be robust in SOL but decreases in EDL, in concordance with earlier findings $[12,13,15]$. Additionally, ISO markedly increased the force $\times$ time integral throughout a prolonged continuous contraction ( $30 \mathrm{~s}$ ) at a high stimulation frequency in SOL (25\%), as shown earlier [14]. Finally, ISO also increased the force $\times$ time integral throughout a prolonged contraction in EDL (10 s), but the increase was small $(<5 \%)$ and appeared to be limited to the first $\mathrm{s}$ of stimulation.

It has been shown that $\beta$-adrenoceptor agonists increase force generation by enhancing SR $\mathrm{Ca}^{2+}$ release (see Introduction) with no noteworthy effect on sensitivity of cross-bridges to $\mathrm{Ca}^{2+}[11,15,30]$. The implication from this finding is that $\beta$-adrenoceptor activation should not alter cross-bridge function via cAMP-dependent protein kinase (PKA) mediated phosphorylation of proteins that affect force generation [11], nor metabolic alterations such as changes in $P_{i}[12]$. With respect to the latter, it is well established that conditions resulting in increased myoplasmic $\mathrm{P}_{\mathrm{i}}$ concentration inhibit force generation, whereas conditions that result in decreases in $P_{i}$ concentration enhance force generation $[1,2]$. Noteworthy is that the inhibition of cross-bridge force generation by $\mathrm{P}_{\mathrm{i}}$ decreases substantially at near physiologic temperature $[23,55]$. At $30^{\circ} \mathrm{C}, \mathrm{P}_{\mathrm{i}}$ has little effect on force at very high $\left[\mathrm{Ca}^{2+}\right]_{\mathrm{i}}(\mathrm{pCa}=5.8)[1]$. However, at lower $\left[\mathrm{Ca}^{2+}\right]_{\mathrm{i}}(\mathrm{pCa}=$ 6.0), the inhibition of force by $\mathrm{P}_{\mathrm{i}}$ becomes marked, even at $30{ }^{\circ} \mathrm{C}$ [1]. Indeed measurements of $\left[\mathrm{Ca}^{2+}\right]_{\mathrm{i}}$ in intact mouse muscle fibers under maximal stimulation conditions (with and without activation of $\beta$-adrenoceptors) demonstrate values that are close to a pCa of $6.0[14,53]$, which are markedly higher than $\mathrm{pCa}$ values observed in frog fibers (i.e., tetanic $\left[\mathrm{Ca}^{2+}\right]_{i}$ is much higher in frog than in mouse fibers) [59]. Thus, changes in myoplasmic $\mathrm{P}_{\mathrm{i}}$ can affect force generation under the conditions of the present study.

Recently, it was shown that administration of terbutaline ( $\beta_{2}$-adrenoceptor agonist) to humans enhanced muscle performance (both mean and peak power output) during all-out cycling over a 10-s period [39]. Biopsies were taken before and after exercise and analyzed for metabolites associated with energy turnover. From the metabolite values reported in the latter study, we estimated the changes in muscle $\mathrm{P}_{\mathrm{i}}$ contents during exercise $(-2[\Delta \mathrm{ATP}]-[\Delta \mathrm{PCr}]-[\Delta$ glucose $6-\mathrm{P}])$. Under placebo conditions, $\mathrm{P}_{\mathrm{i}}$ increased by $40 \mathrm{mmol} / \mathrm{kg}$ dry weight, whereas after inhalation of terbutaline, $\mathrm{P}_{\mathrm{i}}$ increased by only $7 \mathrm{mmol} / \mathrm{kg}$ dry weight. It was suggested that the difference in $\mathrm{P}_{\mathrm{i}}$ could have contributed to the enhanced performance [39]. Further, it was found that terbutaline also significantly increased anaerobic ATP turnover (10\%). These analyses and estimates were based on mixed muscle. The extent to which our findings are applicable to intact humans is unclear. Our results support the idea of lower $\mathrm{P}_{\mathrm{i}}$ and increased ATP turnover as mechanisms to explain and reflect, respectively, enhanced force generation by activation of $\beta$ adrenoceptors during short-term maximal exercise, but it appears that in isolated rodent muscle, decreases in $\mathrm{P}_{\mathrm{i}}$ account for a small enhancement of muscle performance in EDL in response to $\beta_{2}$-adrenoceptor activation. Indeed, conversion of the basal $P_{i}$ contents in EDL to $\mathrm{mM}$ (divide values in Table 3 by three) yield values of $5.6 \mathrm{mM}$ for control and $4.8 \mathrm{mM}$ for ISO. These concentrations would be expected to decrease force to approximately 70 and $80 \%$, respectively, of that measured in the absence of $\mathrm{P}_{\mathrm{i}}$ at $\mathrm{pH} 7.0$ [22]. Our observation that the increase in force in the presence of ISO under maximal stimulation conditions was almost $8 \%$ (Fig. $2 \mathrm{~h}$ ) agrees well the aforementioned results [22]. This implies that ISO enhances force generation by increasing the amount of force generated per cross-bridge in EDL. In SOL, however, the marked enhancement of force generation is associated with a substantial increase in ATP turnover. This likely reflects an increase in the number of active cross-bridges, which can be attributed to an increase in $\left[\mathrm{Ca}^{2+}\right]_{\mathrm{i}}$. Indeed, it appears that under essentially maximal stimulation conditions in SOL, the contractile proteins are not saturated with $\mathrm{Ca}^{2+}$ and therefore terbutaline ( $\beta$-adrenoceptor agonist) administration results in substantial increases in $\left[\mathrm{Ca}^{2+}\right]_{\mathrm{i}}$ as well as force [30]. Therefore, our results are consistent with the latter observation, i.e., the increase in $\left[\mathrm{Ca}^{2+}\right]_{\mathrm{i}}$ would activate more crossbridges and thereby increase ATP turnover.

Direct measurements of $\left[\mathrm{Ca}^{2+}\right]_{i}$ were not performed in the present study. However, an indirect way to assess whether there were substantial differences in $\left[\mathrm{Ca}^{2+}\right]_{\mathrm{i}}$ between ISO and control treatments in the present study would be to examine the phosphorylase data. Previously, Miller [51] provided evidence to support use of phosphorylase activation in isolated perfused rat hearts exposed to epinephrine (and glucagon) to reflect increases in $\left[\mathrm{Ca}^{2+}\right]_{\mathrm{i}}$. Indeed increases in phosphorylase fractional activity in ischemic muscle have been attributed to increases in $\left[\mathrm{Ca}^{2+}\right]_{\mathrm{i}}$, which will activate phosphorylase kinase, resulting in phosphorylation of phosphorylase $b$ (see "Results" and [41]). Thus, one can use changes in phosphorylase fractional activity as an indirect marker of changes in $\left[\mathrm{Ca}^{2+}\right]_{\mathrm{i}}$. First, it should be noted that activation of $\beta$ adrenoceptors does not significantly alter $\left[\mathrm{Ca}^{2+}\right]_{\mathrm{i}}$ in isolated glycolytic or oxidative muscle fibers at rest $[3,15,30]$. ISO induced a marked activation of phosphorylase (increase in fractional activity) in EDL at rest, but there was no additional effect in stimulated muscle (Fig. 4). This is likely explained by near maximal activation of phosphorylase during contraction that would preclude detection of additional activation by ISO. An increase in $\left[\mathrm{Ca}^{2+}\right]_{\mathrm{i}}$ in EDL under this condition in the presence of ISO is unlikely to have a major effect on force production since troponin $\mathrm{C}$ is probably close to saturation with $\mathrm{Ca}^{2+}$ already in the absence of ISO [15]. Indeed, our results show that maximum force and force $\times$ time integral 
are enhanced by ISO only by $\sim 5 \%$. In contrast, ISO significantly increased phosphorylase fractional activity in SOL during the prolonged contraction, suggesting that there was a significant increase in $\left[\mathrm{Ca}^{2+}\right]_{i}$. The latter is consistent with the observation of increases in $\left[\mathrm{Ca}^{2+}\right]_{\mathrm{i}}$ and force in SOL muscle fibers in response to $\beta$-adrenoceptor activation under essentially maximal stimulation conditions $[30,58]$. Indeed, Ha et al. [30] showed that terbutaline increased force and $\left[\mathrm{Ca}^{2+}\right]_{\mathrm{i}}$ by $\sim 30 \%$ at $50 \mathrm{~Hz}$ at $22{ }^{\circ} \mathrm{C}$ in isolated SOL fibers. Our findings that maximal force was increased by $\sim 25 \%$ at $70-100 \mathrm{~Hz}$ in SOL muscle at $30^{\circ} \mathrm{C}$ (Fig. 2) are in good agreement with the latter findings. Therefore, the current phosphorylase results are consistent with earlier direct measurements of $\left[\mathrm{Ca}^{2+}\right]_{\mathrm{i}}$ in SOL fibers, suggesting that $\left[\mathrm{Ca}^{2+}\right]_{\mathrm{i}}$ was elevated in SOL fibers in the present study. Nevertheless, direct measurements of $\left[\mathrm{Ca}^{2+}\right]_{\mathrm{i}}$ under conditions of the present study are required to confirm this.

Another noteworthy observation is that ISO had a greater effect on muscle glycogenolysis in resting EDL than in resting SOL muscle (Table 3). These findings are consistent with earlier observations that showed a preferential activation of glycogenolysis in resting EDL (glycolytic) vs. SOL (oxidative) muscle in response to administration of adrenaline $[17,37,57]$. In contrast, ISO significantly increased lactate accumulation during continuous stimulation in SOL but not in EDL in the present study. This, too, is consistent with earlier findings showing preferential effects of adrenaline on glycogenolysis in oxidative vs. glycolytic fibers during contraction $[27,57]$. In the context of the present study, we propose the following. At rest, energy turnover is low and is not markedly accelerated by $\beta$-adrenoceptor activation. Therefore, the minimal degree of glycogenolysis in EDL at rest (relative to contraction) likely occurs for the purpose of trapping $\mathrm{P}_{\mathrm{i}}$ in hexose phosphates rather than generating ATP. In contrast, the enhancement of glycogenolysis during contraction in SOL primarily occurs to generate more ATP to meet the increased energy demand. Thus, in the context of fight or flight in small mammals, activation of phosphorylase appears to occur for different purposes in oxidative and glycolytic fibers (see below).

Alternative mechanisms to explain the effects of ISO on contractile function under the conditions studied were not investigated in the present study, including activation of $\mathrm{Na}^{+}-\mathrm{K}^{+}$ pumps [20], PKA-dependent phosphorylation of myofilament proteins [49], and trans-sarcolemmal $\mathrm{Ca}^{2+}$ influx [25]. These factors, however, were recently reviewed in detail and it was concluded that they were unlikely to explain the $\beta$ adrenoceptor-dependent enhanced contractile function of isolated skeletal limb muscles [11]. Similarly, it could be argued that soleus fibers have a higher density of $\beta$-adrenoceptors than do EDL fibers [48] and that this accounts for the larger effects of ISO on contractile function in SOL. Although the functional significance of this observation is not fully established, generally the response to $\beta$-agonists is greater in fast- than in slow-twitch skeletal muscle [48]. In the present study, we also observed that the ISO-mediated activation of phosphorylase was larger in EDL than in SOL muscles. Further, administration of supraphysiologic doses of adrenaline results in increases in cAMP content of SOL that are slightly less than that seen in EDL, and the activation of phosphorylase is markedly less in SOL than in EDL [17]. These findings do not support the idea that ISO-enhanced contractile function of SOL derives from increased density of $\beta$ adrenoceptors.

Based on these observations, we propose a three-phase scenario in the fight or flight response of small mammals. (1) Initially, the perception of a predator will result in enhanced sympathetic discharge in the prey. This will serve as a priming effect in preparation for fight or flight (activation of phosphorylase and decrease in $\mathrm{P}_{\mathrm{i}}$ primarily in glycolytic fibers). (2) Attack of the predator will result in a supramaximal effort on the part of the prey to avoid being captured. Here, a small enhancement of force (due to decreased levels of $P_{i}$ in glycolytic fibers at the start of the contraction) could prove critical for survival (e.g., a mouse will jump to avoid a pouncing cat). (3) The final phase is escape by a short sprint to safety (e.g., burrow or hole in a wall). This phase would entail enhanced force generation primarily in oxidative fibers that would last several seconds. Indeed, in support of this idea, activation of motor units in SOL fibers at frequencies approaching $100 \mathrm{~Hz}$ has been documented in freely moving rats [32]. However, for this scenario to be likely, it would require the existence of a sufficient amount of oxidative fibers that can generate adequate power in the active limbs. With respect to the mouse, it appears that $\sim 25-30 \%$ of all hind-limb muscles are accounted for by slow oxidative fibers [10]. However, maximal power output in SOL is only about $30 \%$ of that observed in EDL at temperatures of $20-25^{\circ} \mathrm{C}[6,7,26,47]$. At more physiological temperatures $\left(30-37^{\circ} \mathrm{C}\right)$, this value approaches $60 \%$ [7, 34]. Further, in the fatigued state, maximum power output of EDL decreases by almost $60 \%$, whereas in SOL, it decreases only by $\sim 25 \%$ [4]. Taken together, these data suggest that the proposed three-phase scenario is feasible.

In conclusion, under conditions of maximal stimulation, ISO has a minor positive effect on force generation in EDL that is associated with a decrease in $\mathrm{P}_{\mathrm{i}}$ content during onset of contraction. In contrast, ISO has a marked enhancing effect on force generation in SOL that is associated with an increase in glycogenolysis and ATP turnover. Thus ISO-mediated activation of phosphorylase contributes to enhanced performance of SOL and EDL muscles via separate mechanisms.

Acknowledgements Open access funding provided by Swedish School of Sport and Health Sciences (GIH). 
Author contributions All experiments were performed in the Muscle Research Laboratory at Ariel University. A.K. conceived and designed the research; S.J.B. performed the experiments; S.J.B. analyzed the data; S.J.B. and A.K. interpreted results of experiments; S.J.B. and A.K. prepared figures; S.J.B. and A.K. edited and revised the manuscript; S.J.B. and A.K. approved the final version of the manuscript; S.J.B. and A.K. drafted the manuscript.

Funding information This research was supported by a grant from the Israel Science Foundation (no. 870/15).

\section{Compliance with ethical standards}

Conflict of interest The authors declare that they have no conflict of interest.

Open Access This article is distributed under the terms of the Creative Commons Attribution 4.0 International License (http:// creativecommons.org/licenses/by/4.0/), which permits unrestricted use, distribution, and reproduction in any medium, provided you give appropriate credit to the original author(s) and the source, provide a link to the Creative Commons license, and indicate if changes were made.

\section{References}

1. Allen D, Trajanovska S (2012) The multiple roles of phosphate in muscle fatigue. Front Physiol 3. https://doi.org/10.3389/fphys. 2012.00463

2. Allen DG, Lamb GD, Westerblad H (2008) Skeletal muscle fatigue: cellular mechanisms. Physiol Rev 88:287-332. https://doi.org/10. 1152/physrev.00015.2007

3. Andersson DC, Betzenhauser MJ, Reiken S, Umanskaya A, Shiomi T, Marks AR (2012) Stress-induced increase in skeletal muscle force requires protein kinase a phosphorylation of the ryanodine receptor. J Physiol 590:6381-6387. https://doi.org/10.1113/ jphysiol.2012.237925

4. Barclay CJ (1996) Mechanical efficiency and fatigue of fast and slow muscles of the mouse. J Physiol 497(Pt 3):781-794

5. Barclay CJ (2005) Modelling diffusive O2 supply to isolated preparations of mammalian skeletal and cardiac muscle. J Muscle Res Cell Motil 26:225-235. https://doi.org/10.1007/s10974-005-9013$\mathrm{x}$

6. Barclay CJ, Constable JK, Gibbs CL (1993) Energetics of fast- and slow-twitch muscles of the mouse. J Physiol 472:61-80

7. Barclay CJ, Woledge RC, Curtin NA (2010) Is the efficiency of mammalian (mouse) skeletal muscle temperature dependent? J Physiol 588:3819-3831. https://doi.org/10.1113/jphysiol.2010. 192799

8. Bonen A, Megeney LA, McCarthy SC, McDermott JC, Tan MH (1992) Epinephrine administration stimulates GLUT4 translocation but reduces glucose transport in muscle. Biochem Biophys Res Commun 187:685-691. https://doi.org/10.1016/0006-291X(92) 91249-P

9. Bowman WC, Nott MW (1969) Actions of sympathomimetic amines and their antagonists on skeletal muscle. Pharmacol Rev 21:27-72

10. Burkholder TJ, Fingado B, Baron S, Lieber RL (1994) Relationship between muscle fiber types and sizes and muscle architectural properties in the mouse hindlimb. J Morphol 221:177-190. https://doi. org/10.1002/jmor. 1052210207

11. Cairns SP, Borrani F (2015) Beta-adrenergic modulation of skeletal muscle contraction: key role of excitation-contraction coupling. J Physiol 593:4713-4727. https://doi.org/10.1113/JP270909
12. Cairns SP, Dulhunty AF (1993) Beta-adrenergic potentiation of E-C coupling increases force in rat skeletal muscle. Muscle Nerve 16: 1317-1325. https://doi.org/10.1002/mus.880161208

13. Cairns SP, Dulhunty AF (1993) The effects of $\beta$-adrenoceptor activation on contraction in isolated fast- and slow-twitch skeletal muscle fibres of the rat. Br J Pharmacol 110:1133-1141. https:// doi.org/10.1111/j.1476-5381.1993.tb13932.x

14. Cairns SP, Dulhunty AF (1994) Beta-adrenoceptor activation shows high-frequency fatigue in skeletal muscle fibers of the rat. Am J Phys 266:C1204-C1209. https://doi.org/10.1152/ajpcell.1994.266. 5.C1204

15. Cairns SP, Westerblad H, Allen DG (1993) Changes of tension and $[\mathrm{Ca} 2+] \mathrm{i}$ during beta-adrenoceptor activation of single, intact fibres from mouse skeletal muscle. Pflugers Arch - Eur J Physiol 425: $150-155$

16. Cannon WB (1929) Bodily changes in pain, hunger, fear and rage. Appleton, Oxford

17. Chasiotis D (1985) Effects of adrenaline infusion on cAMP and glycogen phosphorylase in fast-twitch and slow-twitch rat muscles. Acta Physiol Scand 125:537-540

18. Chiasson J-L, Shikama H, Chu DTW, Exton JH (1981) Inhibitory effect of epinephrine on insulin-stimulated glucose uptake by rat skeletal muscle. J Clin Invest 68:706-713. https://doi.org/10.1172/ JCI110306

19. Clark MG, Patten GS (1984) Adrenergic regulation of glucose metabolism in rat heart. A calcium-dependent mechanism mediated by both alpha- and beta-adrenergic receptors. J Biol Chem 259:15204 15211

20. Clausen T, Flatman JA (1977) The effect of catecholamines on Na$\mathrm{K}$ transport and membrane potential in rat soleus muscle. J Physiol 270:383-414

21. Cooke R, Pate E (1985) The effects of ADP and phosphate on the contraction of muscle fibers. Biophys J 48:789-798

22. Crow MT, Kushmerick MJ (1982) Chemical energetics of slowand fast-twitch muscles of the mouse. J Gen Physiol 79:147-166

23. Debold EP, Romatowski J, Fitts RH (2006) The depressive effect of $\mathrm{Pi}$ on the force-pCa relationship in skinned single muscle fibers is temperature dependent. Am J Physiol Cell Physiol 290:C1041C1050. https://doi.org/10.1152/ajpcell.00342.2005

24. Fujimura N, Sumita S, Narimatsu E, Nakayama Y, Shitinohe Y, Namiki A (2000) Effects of isoproterenol on diaphragmatic contractility in septic peritonitis. Am J Respir Crit Care Med 161:440 446. https://doi.org/10.1164/ajrccm.161.2.9904044

25. Garcia J, Gamboa-Aldeco R, Stefani E (1990) Charge movement and calcium currents in skeletal muscle fibers are enhanced by GTP gamma S. Pflugers Arch 417:114-116

26. Graber TG, Kim JH, Grange RW, McLoon LK, Thompson LV (2015) C57BL/6 life span study: age-related declines in muscle power production and contractile velocity. Age (Dordr) 37:9773. https://doi.org/10.1007/s11357-015-9773-1

27. Greenhaff PL, Ren JM, Soderlund K, Hultman E (1991) Energy metabolism in single human muscle fibers during contraction without and with epinephrine infusion. Am J Phys 260:E713-E718. https://doi.org/10.1152/ajpendo.1991.260.5.E713

28. Greenhaff PL, Soderlund K, Ren JM, Hultman E (1993) Energy metabolism in single human muscle fibres during intermittent contraction with occluded circulation. J Physiol 460:443-453

29. Gross SR, Mayer SE, Longshore MA (1976) Stimulation of glycogenolysis by beta adrenergic agonists in skeletal muscle of mice with the phosphorylase kinase deficiency mutation (I strain). J Pharmacol Exp Ther 198:526-538

30. Ha TN, Posterino GS, Fryer MW (1999) Effects of terbutaline on force and intracellular calcium in slow-twitch skeletal muscle fibres of the rat. Br J Pharmacol 126:1717-1724. https://doi.org/10.1038/ sj.bjp. 0702482 
31. Hayes JS, Bowling N, Boder GB (1984) Contractility and protein phosphorylation in cardiomyocytes: effects of isoproterenol and AR-L57. Am J Phys 247:H157-H169. https://doi.org/10.1152/ ajpheart.1984.247.2.H157

32. Hennig R, Lømo T (1985) Firing patterns of motor units in normal rats. Nature 314:164-166. https://doi.org/10.1038/314164a0

33. Hickson RC (1981) Skeletal muscle cytochrome c and myoglobin, endurance, and frequency of training. J Appl Physiol Respir Environ Exerc Physiol 51:746-749. https://doi.org/10.1152/jappl. 1981.51.3.746

34. Higgins MF, Tallis J, Price MJ, James RS (2013) The effects of elevated levels of sodium bicarbonate $(\mathrm{NaHCO}(3))$ on the acute power output and time to fatigue of maximally stimulated mouse soleus and EDL muscles. Eur J Appl Physiol 113:1331-1341. https://doi.org/10.1007/s00421-012-2557-8

35. Hostrup M, Kalsen A, Bangsbo J, Hemmersbach P, Karlsson S, Backer V (2014) High-dose inhaled terbutaline increases muscle strength and enhances maximal sprint performance in trained men. Eur J Appl Physiol 114:2499-2508. https://doi.org/10.1007/ s00421-014-2970-2

36. Ivy JL, Chi MM, Hintz CS, Sherman WM, Hellendall RP, Lowry OH (1987) Progressive metabolite changes in individual human muscle fibers with increasing work rates. Am J Phys Cell Phys 252:C630-C639. https://doi.org/10.1152/ajpcell.1987.252.6.C630

37. Jensen J, Dahl HA, Opstad PK (1989) Adrenaline-mediated glycogenolysis in different skeletal muscle fibre types in the anaesthetized rat. Acta Physiol Scand 136:229-233. https://doi.org/10.1111/ j.1748-1716.1989.tb08656.x

38. Jordan J, Tank J, Stoffels M, Franke G, Christensen NJ, Luft FC, Boschmann M (2001) Interaction between $\beta$-adrenergic receptor stimulation and nitric oxide release on tissue perfusion and metabolism. J Clin Endocrinol Metab 86:2803-2810. https://doi.org/10. 1210/jcem.86.6.7567

39. Kalsen A, Hostrup M, Soderlund K, Karlsson S, Backer V, Bangsbo J (2016) Inhaled Beta2-agonist increases power output and glycolysis during sprinting in men. Med Sci Sports Exerc 48:39-48. https://doi.org/10.1249/mss.0000000000000732

40. Kammoun M, Cassar-Malek I, Meunier B, Picard B (2014) A simplified immunohistochemical classification of skeletal muscle fibres in mouse. Eur J Histochem 58:2254. https://doi.org/10.4081/ejh. 2014.2254

41. Katz A (1997) Differential responses of glycogen synthase to ischaemia and ischaemic contraction in human skeletal muscle. Exp Physiol 82:203-211

42. Katz A, Andersson DC, Yu J, Norman B, Sandstrom ME, Wieringa B, Westerblad H (2003) Contraction-mediated glycogenolysis in mouse skeletal muscle lacking creatine kinase: the role of phosphorylase b activation. J Physiol 553:523-531. https://doi.org/10.1113/ jphysiol.2003.051078

43. Katz A, Hernández A, Caballero DMR, Briceno JFB, Amezquita LVR, Kosterina N, Bruton JD, Westerblad H (2014) Effects of Nacetylcysteine on isolated mouse skeletal muscle: contractile properties, temperature dependence, and metabolism. Pflugers Arch Eur J Physiol 466:577-585. https://doi.org/10.1007/s00424-0131331-z

44. Leclerc N, Ribera F, Zoll J, Warter JM, Poindron P, Lampert E, Borg J (2001) Selective changes in mitochondria respiratory properties in oxidative or glycolytic muscle fibers isolated from G93AhumanSOD1 transgenic mice. Neuromuscul Disord 11: $722-727$

45. Lowry OH, Passonneau JV (1972) A flexible system of enzymatic analysis. Academic Press, New York

46. Luedeke JD, McCall RD, Dillaman RM, Kinsey ST (2004) Properties of slow- and fast-twitch skeletal muscle from mice with an inherited capacity for hypoxic exercise. Comp Biochem Physiol A Mol Integr Physiol 138:373-382. https://doi.org/10.1016/j.cbpb. 2004.05.010

47. Lynch GS, Hinkle RT, Chamberlain JS, Brooks SV, Faulkner JA (2001) Force and power output of fast and slow skeletal muscles from mdx mice 6-28 months old. J Physiol 535:591-600

48. Lynch GS, Ryall JG (2008) Role of beta-adrenoceptor signaling in skeletal muscle: implications for muscle wasting and disease. Physiol Rev 88:729-767. https://doi.org/10.1152/physrev.00028. 2007

49. Matsuba D, Terui T, O-Uchi J, Tanaka H, Ojima T, Ohtsuki I, Ishiwata S, Kurihara S, Fukuda N (2009) Protein kinase Adependent modulation of $\mathrm{Ca} 2+$ sensitivity in cardiac and fast skeletal muscles after reconstitution with cardiac troponin. J Gen Physiol 133:571-581. https://doi.org/10.1085/jgp.200910206

50. Millar NC, Homsher E (1990) The effect of phosphate and calcium on force generation in glycerinated rabbit skeletal muscle fibers. A steady-state and transient kinetic study. J Biol Chem 265:20234 20240

51. Miller TB Jr (1984) Phosphorylase activation hypersensitivity in hearts of diabetic rats. Am J Phys 246:E134-E140. https://doi.org/ 10.1152/ajpendo.1984.246.2.E134

52. Mishra P, Varuzhanyan G, Pham AH, Chan DC (2015) Mitochondrial dynamics is a distinguishing feature of skeletal muscle fiber types and regulates organellar compartmentalization. Cell Metab 22:1033-1044. https://doi.org/10.1016/j.cmet.2015.09.027

53. Nielsen J, Cheng AJ, Ørtenblad N, Westerblad H (2014) Subcellular distribution of glycogen and decreased tetanic $\mathrm{Ca}(2+)$ in fatigued single intact mouse muscle fibres. J Physiol 592:20032012. https://doi.org/10.1113/jphysiol.2014.271528

54. Ortenblad N, Macdonald WA, Sahlin K (2009) Glycolysis in contracting rat skeletal muscle is controlled by factors related to energy state. Biochem J 420:161-168. https://doi.org/10.1042/ bj20082135

55. Pate E, Bhimani M, Franks-Skiba K, Cooke R (1995) Reduced effect of $\mathrm{pH}$ on skinned rabbit psoas muscle mechanics at high temperatures: implications for fatigue. J Physiol 486(Pt 3):689-694

56. Reading SA, Murrant CL, Barclay JK (2003) Increased cAMP as a positive inotropic factor for mammalian skeletal muscle in vitro. Can J Physiol Pharmacol 81:986-996. https://doi.org/10.1139/ y03-104

57. Richter EA, Ruderman NB, Gavras H, Belur ER, Galbo H (1982) Muscle glycogenolysis during exercise: dual control by epinephrine and contractions. Am J Physiol Endocrinol Metab 242:E25-E32. https://doi.org/10.1152/ajpendo.1982.242.1.E25

58. Segal SS, Faulkner JA, White TP (1986) Skeletal muscle fatigue in vitro is temperature dependent. J Appl Physiol 61:660-665. https://doi.org/10.1152/jappl.1986.61.2.660

59. Westerblad H, Lannergren J, Allen DG (1997) Slowed relaxation in fatigued skeletal muscle fibers of Xenopus and mouse. Contribution of $\left[\mathrm{Ca}^{2+}\right] \mathrm{i}$ and cross-bridges. J Gen Physiol 109:385-399

60. Young DA, Wallberg-Henriksson H, Cranshaw J, Chen M, Holloszy JO (1985) Effect of catecholamines on glucose uptake and glycogenolysis in rat skeletal muscle. Am J Phys 248:C406C409

61. Zhang SJ, Andersson DC, Sandstrom ME, Westerblad H, Katz A (2006) Cross bridges account for only $20 \%$ of total ATP consumption during submaximal isometric contraction in mouse fast-twitch skeletal muscle. Am J Physiol Cell Physiol 291:C147-C154. https://doi.org/10.1152/ajpcell.00578.2005

Publisher's note Springer Nature remains neutral with regard to jurisdictional claims in published maps and institutional affiliations. 